\title{
Implementation of an Open Network Infrastructure for Internet of Things based on LoRaWAN
}

\author{
Jamile Leite and Leonardo Ramalho
}

\begin{abstract}
This manuscript shows the development of an open infrastructure that aims to be free and available for anyone interested in developing IoT applications. The idea is that users can use the infrastructure without concerning about the details of the IoT network. This manuscript shows some details of the infrastructure implementation and the provided documentation.
\end{abstract}

\section{Keywords-IoT, LPWAN, LoRaWAN.}

\section{INTRODUCTION}

A huge rise in the number of objects connected to the internet, either by wire or wireless has made Internet of Things (IoT) an increasingly growing topic of conversation both in the industry and academy. In particular, IoT is a concept that has the potential to impact many aspects of industry and out lives. It can dramatically improve security, energy efficiency, decision-making and productivity of enterprises in retail, supply chain management, manufacturing, agriculture, education, health, and many other aspects of daily life for consumers, through amazing solutions [1].

There are various technologies suitable for IoT networks [2], but the Low Power Wide Area Networks (LPWAN) are particularly important because they provide long range and massive scale connectivity for low power, low cost, and low data rate IoT devices [3]. In this context, two LPWAN technologies that operate on unlicensed frequencies are predominant: Long Range Wide Area Network (LoRaWAN) and Sigfox. The former uses a closed chipset for the physical layer called LoRa, but provides an open network, while the latter uses a closed network but freely provides the physical layer protocol specification to chip manufacturers (i.e., open chipset) [4].

Based on the above characteristics, we use LoRaWAN to implement an open and free IoT network infrastructure. More specifically, the LoRaWAN can operate on unlicensed frequencies and the network can be operated with open software or free services. Furthermore, a single LoRaWAN gateway can serve multiples nodes and cover an area with some kilometers in radius. All these features allow the deployment of an IoT network at a low cost.

This paper details the design of the construction of a LoRaWAN network infrastructure and its dissemination through the elaboration of technical documentation [5] describing the network and how to use it. The idea of this proposal is that the created infrastructure and the supporting documentation generated in the project can facilitate the development of

The authors would like to thank PROPESP/UFPA for the support offered via PRODOUTOR2020 program.

Jamile Leite and Leonardo Ramalho are with Computer and Telecommunications Engineering Department at Federal University of Para (UFPA), Belem-PA 66615-170, Brazil (e-mails: jamile.leite@itec.ufpa.br and leonardolr@ufpa.br).

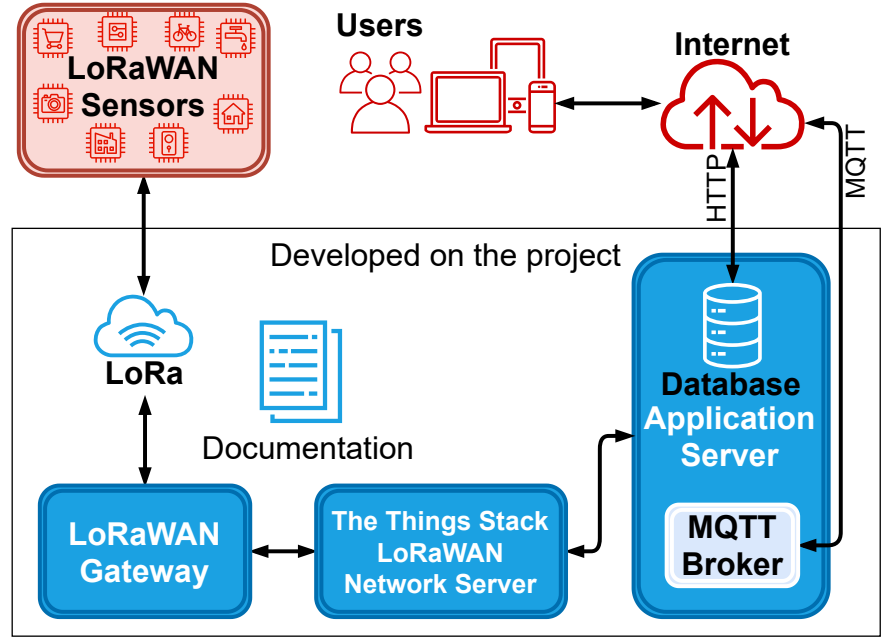

Fig. 1. Implemented IoT Network Infrastructure based on LoRaWAN.

IoT applications; so that anyone interested in developing IoT applications can be mainly concerned with the application or the devices, and use the IoT network without costs.

The documentation made available shows how the users can connect theirs devices to the network and how to access the data. Furthermore, the documentation may help others build new IoT networks, or even expand the network infrastructure, for example, by installing new gateways, which would help to improve service coverage and availability. With this, the project could help to popularize local solutions that are based on the Internet of Things, facilitating their development by the academic community and other sectors of society.

\section{IMPLEMENTED IoT ARCHITECTURE}

The main components of a LoRaWAN network are the sensor devices, the gateway, the network server and the application server. More details about the function of each LoRaWAN component can be found in [3].

In this work, the LoRaWAN network was implemented using the gateway RG191 from Laird's Sentrius [6], while the network server and application server were implemented with The Things Stack (TTS) Community Edition [7] from The Things Network. Fig. 1 shows the architecture of the deployed network, where TTS provides a database and an MQTT broker. Thus, the users can access the data of their devices by using HyperText Transfer Protocol (HTTP) or Message Queuing Telemetry Transport (MQTT) protocol. The open network infrastructure for IoT based on LoRaWAN developed in this project is named "UFPIoT".

The proposed architecture is not novel. However, this manuscript presents the constructions of the LoRaWAN net- 
work and makes its usage transparent to the user, i.e., the user does not need to be concerned about the details of the implementation of the infrastructure.

The Things Network is a global collaborative Internet of Things ecosystem that creates networks, devices and solutions using LoRaWAN. The Things Network runs the TTS Community Edition, which is a crowdsourced, open and decentralized LoRaWAN network. It supports all LoRaWAN classes (A, B, C) and multicast device groups, all existing LoRaWAN versions (including v1.0.4 and v1.1) and all regional parameters as defined by the LoRa Alliance ${ }^{\circledR}$ [8]. The Things Stack is developed and maintained by The Things Industries [7].

TTS's application server exposes an MQTT broker to work with streaming events, allowing connected MQTT clients to subscribe to uplinks from the desired devices or publish downlinks to them. TTS has also a Storage Integration that allows storing received upstream messages in a persistent database, and retrieving them at a later time using its HTTP Application Programming Interface (API). Both resources were configured in this network's application server to be available to users.

\section{DOCUMENTATION}

The steps and settings for connecting an end-device to the network, with full access to the MQTT and storing features, are described in the provided teaching material [5] with the necessary keys and passwords for connection and authentication. To add a LoRaWAN node to network, information provided by the manufacturer of the end-device has to be set when adding it to the application server. This information is related to the device's unique identifiers and the LoRaWAN specifications it conforms to: LoRaWAN version (all versions), LoRaWAN class capabilities (class A, B and C), frequency sub-band, regional parameters and their version (PHY v1.0.2 REV A or B). The frequency plan accepted is the US 902$928 \mathrm{MHz}$ due to gateway configuration. The user should also provide a device ID (devID) for identification of the device in the application.

For Over The Air Activation (OTAA), application EUI (AppEUI), device EUI (DevEUI) and application key (AppKey) are also necessary device's identifiers for the connection. They are often provided by manufacturer, but can be assigned by TTS or created by user.

For Activation By Personalisation (ABP) and Multicast, network session key (NwkSKey) and AppKey are required. They are generally provided by manufacturer, but can also be issued by TTS as the device address (DevAddr) that is provided to the user. If Class B is enabled (for any activation mode), it is necessary ping slot periodicity and frequency.

After the configurations are done in the application server, the device uses its identifiers to connect to the server. It is ready to send and receive data through the LoRaWAN network.

To receive the data sent by the device and send messages to it, the user has to connect to the server's MQTT Broker as a client with an MQTT API key. Two topics are made available: one to subscribe, where the client receives all uplink messages, and one to publish, where the client schedules downlink messages. API key and topics are provided in the documentation, with examples for publish and subscribe in Mosquitto[9] and Node.js.

The data received through MQTT are JavaScript Object Notation (JSON) objects. The JSON object contains IDs related to the end device and its connection to the server (DevId, DevEUI, DevAddr, Application ID, gateway ID), correlation IDs, timestamp and the uplink message with metadata sent from the device, including the payload, a counter, datarate, spreading factor, coding rate, frequency and consumed airtime. The raw payload comes in base64 format.

The application's database contains received upstream messages that can be retrieved by accessing its end-points with an API key through an HTTP connection. API key and endpoints are provided in the documentation. The documents have examples using a web navigator and Windows command line.

The data contained in the database are JSON objects with only one key named "results", whose value is another JSON object with the same format of the one that is received through an MQTT connection.

Since The Things Stack Community is crowdsourced, any user in the gateway coverage will also be able to create their own application server in the network server but from scratch and without the configurations and features already set in the one built for this project.

\section{CONCLUSiOnS}

This paper presented the development of an open IoT network based on LoRaWAN and its technical documentation. The project aims to facilitate the development of IoT applications by the use of the created infrastructure and supporting material, where the developer can be mainly concerned with the sensor nodes and the application to be deployed. With the documentation developed in this project, the user can easily connect to LoRaWAN, have access to the device and its data, and abstract the network configurations while using the IoT network without cost.

\section{REFERENCES}

[1] N. Sharma, M. Shamkuwar, and I. Singh, The History, Present and Future with IoT, V. E. Balas, V. K. Solanki, R. Kumar, and M. Khari, Eds. Cham: Springer International Publishing, 2019. [Online]. Available: https://doi.org/10.1007/978-3-030-04203-5

[2] J. Ding, M. Nemati, C. Ranaweera, and J. Choi, "IoT Connectivity Technologies and Applications: A Survey," IEEE Access, vol. 8, pp. $67646-67673,2020$.

[3] W. Ayoub et al., "Internet of Mobile Things: Overview of LoRaWAN, DASH7, and NB-IoT in LPWANs Standards and Supported Mobility,' IEEE Comm. Surveys Tutorials, vol. 21, no. 2, pp. 1561-1581, 2019.

[4] A. Ikpehai et al., "Low-Power Wide Area Network Technologies for Internet-of-Things: A Comparative Review," IEEE Internet of Things Journal, vol. 6, no. 2, pp. 2225-2240, 2019.

[5] J. Leite and L. Ramalho, "Tutorial on how to use the UFPIoT network," https://docs.google.com/document/d/1U_NaDKDjkiYKrFnstjNQTmg3uezP0QPFpCI3taUNw, accessed: 2021-05-01.

[6] "Laird Connectivity RG191 Datasheet," https://www.lairdconnect.com/documentation/product-brief-sentriusrg1xx-series-gateway, accessed: 2021-04-30.

[7] The Things Industry, "What Is The Things Stack?" https://www.thethingsindustries.com/docs/getting-started/what-is-tts, accessed: 2021-05-01.

[8] L. Alliance, "About the LoRaWAN® Specification," https://loraalliance.org/lorawan-for-developers/, accessed: 2021-05-01.

[9] R. A. Light, "Mosquitto: server and client implementation of the mqtt protocol," Journal of Open Source Software, vol. 2, no. 13, p. 265, 2017. 